\title{
A Novel Method for Night-Time Single Image Dehazing
}

\author{
Prince Owusu-Agyeman, Xie Wei, James Okae \\ School of Automation Science and Engineering, South China University of Technology, Guangzhou, China \\ Email: Pagyeman@qq.com,weixie@scut.edu.cn, jamesokae@yahoo.com
}

How to cite this paper: Owusu-Agyeman, P., Wei, X. and Okae, J. (2019) A Novel Method for Night-Time Single Image Dehazing. Journal of Computer and Communications, 7, 76-87.

https://doi.org/10.4236/jcc.2019.711006

Received: October 8, 2019

Accepted: November 17, 2019

Published: November 20, 2019

Copyright $\odot 2019$ by author(s) and Scientific Research Publishing Inc. This work is licensed under the Creative Commons Attribution International License (CC BY 4.0).

http://creativecommons.org/licenses/by/4.0/

\begin{abstract}
Images acquired under deprived weather environment are frequently corrupted due to the presence of haze, mist, fog or other aerosols in a form of noise. Haze elimination is essential in computer vision and computational photography applications. Generally, there is the existence of numerous approaches towards haze removal which are mostly meant for hazy images under daytime environments. Although the potency of these proposed approaches has been comprehensively established on daylight hazy images. However these procedures inherit significant limitations on images influenced by night-time hazy environments. Since night time haze removal dehazing remains an ill-posed problem, we proposed a novel method for night-time single image dehazing which is efficient under night-time environments. The proposed scheme is a dark channel-based local image dehazing procedure that locally estimates the atmospheric intensity for each selected mask on a corrupted image independently and not the entire image. This is done in order to overcome the challenge of night-scenes that are exposed to multiple/artificial lights source and spatially non-uniform environmental illumination. We performed an adaptive filtering on the combined dehazed masks to improve the degraded image. We validated the supremacy of the proposed approach in terms of speed and robustness through computer-based experiments. Conclusively, we displayed comparison results with state-of-the-art and extensively emphasized the comparative advantage of our scheme.
\end{abstract}

\section{Keywords}

Nighttime, Haze Removal, Dark Channel Prior, Image Enhancement, Machine Vision

\section{Introduction}

Outdoor images acquired under poor weather are frequently corrupted due to the presence of haze mist, fog or other aerosols as noise. This is a result of the 
absorption and the scattering of light that travels from the point or scene of interest to the acquisition device caused by the existence of aerosols thus direct attenuation. Simultaneously, the presence of the aerosols also scatters the airlight through the transmission from the point of interest to the observer, subsequently leads to color distortions, inferior visibility and low contrast on captured images. However such factors make it an ill-posed challenge of capturing clear image under deprived environment. Numerous techniques of haze removal in computer vision and image processing have been established over the last decades. These techniques encompass various procedures used to retrieve information such as contrast extraction, feature extraction, scene depth, color channels and others. The corruption process associated with images acquired under hazy environment has been well recognized by computer vision and computer graphics in some existing works [1] [2] [3] [4], where the degradation process can be formulated as:

$$
I(x)=J(x) t(x)+(1-t(x)) A
$$

where $I$ denotes the intensity observed of the haze image. $x$ represents pixel's index. $J$ represents the scene radiance of the haze-free image which is anticipated to be attained by dehazing procedures. $t$ represents transmission medium which is the part of light that does not scatter and reaches the observer. $A$ represents the global atmospheric light. The main objective of haze removal is to recover $J$, $A$ and $t$ from $I$. Under day-time hazy images, the atmospheric light is primarily established by sky light and incidental sunlight that has been scattered by aerosols or clouds.

In computer vision, graphic systems and other related applications, image corruption persistently continues to be a significant challenge to be resolved. Outdoor systems such as traffic monitoring [5], autonomous self-driving cars [6], outdoor security and safety surveillance [7] are commonly affected by image corruption due to adverse weather conditions. Under such uncontrolled weather or environment of outdoor systems, the image acquisition process is certainly influenced adverse weather and the aerosol such as haze, fog, smoke and other atmospheric particles. For this reason several dehazing methods have been established to deal with haze removal by applying multiple images or additional information. For instance, images using diverse degrees of polarization [8], multiple images can be used to estimate object depth under different weather conditions as proposed in [9]. Or image of the scene in addition to the corrupted image [10]. With this challenge of multiple images requirement to acquire desired images, researchers over the last decade proposed single image-based haze removal using prior knowledge and additional depth information [2] [3] [4]. Usually, most of these procedures are suitable in tackling day-time haze removal from images captured under haze environments. Day time algorithms inherit uniform illumination influenced by the skylight hence most daytime dehazing algorithms estimate the atmospheric intensity globally over the entire image. However, the performances of such methods are not impressive under night-time hazy envi- 
ronment due to existence of multiple light sources and intrinsic non-uniform illumination makes difficult to estimate the atmospheric light globally. The work in this paper is to propose a novel night time single dehazing method to restore images that are corrupted under night time hazy conditions. The remainder of this paper includes Section 2 research background, Section 3 presents the proposed methodology, Section 4 presents the experimental evaluations of the proposed scheme with the state of art. Conclusions as well as discussion are drawn in Section 5.

\section{Background}

Generally, images taken under night time conditions are mostly influenced by low natural illumination and non-uniform artificial light or incident light as a result of the existence multiple light source which usually unveil some poor properties like low overall brightness and non-uniform illumination. In addition, the haze may degrade the image quality for its scattering and attenuation effects [11]. Consequently, images under such conditions are normally affected by low contrast and data loss. Additionally, the color of artificial light source affects the dehazing results and the quality of the recovered image. Generally, haze removal process under hazy environment, the atmospheric scattering model has introduced some form of reputable patterns of decomposing and formulating the haze formation process. Primarily as proposed in [11] and advanced works over the years in [1] and [9], the atmospheric scattering model is generally formulated as denoted in the (1). In (1), $t(x)$ denotes the portion of light that reaches to the acquisition device and can be expressed as:

$$
t(x)=\mathrm{e}^{(-\beta d(x))}
$$

where $d(x)$ denotes the distance between portion of interest in the target haze scene and the camera. $\beta$ denotes the scattering coefficient of the medium. Although the formulation of (2) proposes that the distance, $d(x)$ yields $A=I(x)$ as it moves towards infinity, practical circumstances compel this distance to albeit large, real numbers. This distance inherits an inverse correlation with the transmission parameter, in this manner demonstrating one of the key challenges in image dehazing processes; effectively improving image features within distant scene areas. In general, when the atmospheric scattering coefficient $A$, and the scene transmission $t$ are determined, the factual scene is attainable and can be expressed as:

$$
J=\frac{1-A}{t}+A
$$

Tan [2] proposed a procedure to tackle the challenge of corrupted image by increasing the image contrast in a spatially consistent way. This is inspired by the observation that hazy-free images have extra contrast than the hazy ones. Since it assumes the atmospheric intensity is globally uniform, it may fail under night time condition due to the presence of incident lights. Besides, the recovered images have irregular edges. In applications like target recognition systems, 
where edge information are significant, this method can compromise recognition. He et al. [3] established a dark-channel-prior technique to estimate the transmission map. The dark channel prior specifies that normally outdoor images comprise of some pixels with low intensities which are closer to zero in at least one of color channels. This procedure employs the soft matting technique for images restoration. Although this approach is satisfactory yet it is not impressive on night time hazy images due to the effects of incident lights under such conditions the global estimation of the atmospheric light over the entire image is invalid. Besides, it is not real-time feasible due to delays in the construction of dark channel images. Fattal's et al. [4] proposed a method established on the notion that the surface shading and optical transmission are uncorrelated statistically and locally. This method relies on physical features and attains good results on day time scenarios. The performance of this technique under night time conditions is not impressive since it assumes is recognized and does not estimate it explicitly. However, the performance significantly relies on the quality of the input image. Fattal's procedure may be less efficient when there is a presence in the absence of multiplicative variant in substantial parts of the image. Besides, the procedure is slow.

Tang et al. [12] established a dehazing procedure through a learning framework. This technique assumes that transmittance is independent of scene content and within a small patch it is constant. This scheme is applied to synthetically construct hazy patches with several transmittance from haze-free natural image patches. At that point, a regression model is learned from this data to calculate transmittance. However, the procedure demonstrates color shifts due to the spatially varying lights present in night time hazy scenes. Tarel et al. [13] established an innovative procedure and variations of it for restoring scene visibility. The core benefit of this technique is its swiftness, which is the first method to allow visibility restoration in real-time domain. The procedure's intricacy relies linearly on the number of pixels in the image. However, the dehazing results demonstrate abnormal coloring or color distortion with some haze at the edges of the images. Gibson et al. [14] proposed a technique established on dark channel prior by applying a median filter as a substitute of a minimum filter, this technique is swift in recovering images. Besides, the restored images inherits low luminance and dark halos. Liu et al. [15] proposed a technique where a parameter that can automatically regulate the amount of haze required to be removed. Though it has the advantage of fast processing, the restored images are associated with color distortions. Perhaps aside prior-based feature methods, one of key impact in the single image dehazing are the Retinex-based Theory [16]. This is based on the main assumption that in any given image can be divided into reflectance and illumination. These assumptions present the fundamentals on which the schemes of image dehazing are capable of regulating the observed quality of the image. Some of the extended works in relation to this theory are the Single-Scale Retinex (SSR) [17] and the Multi-Scale Retinex (MSR) [18]. Although the potency of these proposed approaches has been comprehensively es- 
tablished on daylight hazy images, they inherit significant limitations on images under night-time hazy scenes. Image haze removal tends to be challenging under night time conditions due factors such as multiple light source in the haze image, inadequate brightness information of haze image and in some cases the presence of different artificial light colors causing non-uniform illumination. Since night time haze removal dehazing remains an ill-posed problem, researchers has express a lot of interest in this domain in order to solve this challenge. Quite a few methods have been proposed specifically for night time dehazing conditions, Pei et al. [19] proposed night-time image dehazing method that exploits the same imaging model applied on daytime dehazing and introduced a preprocessing phase. This color transfer preprocessing phase attempts to solve the color unfairness due to the presence of artificial light by altering the color information to that of a target image. Then a modified dark channel prior is applied to dehaze the image followed by local contrast enhancement via bilateral filtering which results in an absolute grayish scene. However, the output of this method may be different from the expected illumination balanced one and will affect the final dehazed result. Zhang et al. [20] established a new imaging model to account for spatially varying atmospheric light. Their preprocessing phase compensates the incident light intensity by applying Retinex technique and also improves the colors of incident light before applying dark channel prior for dehazing. This method is a relaxed model, which estimates the atmospheric light using local neighborhood instead of computing it globally. However, dehazing results demonstrates obvious glow effects in the restored image. Li et al. [21] proposed a method of haze removal of night-time images by reducing the halos caused by multiple scattering of light near the light sources. Meng et al. [22] proposed a method based on the color transfer theory, where the illumination level of nighttime hazy image can be artificially improved through adaptably choosing the reference image, in divergence to the classical model of color transfer with the approach of overall to overall transfer. The modified model highlights on the diverse features of various areas on the original image. Besides it performs perfectly even though the nighttime image is restricted by the existence of numerous artificial light sources. Moreover, [22] enhanced dehazing scheme is supported and based on the theory of guided image filtering which is implemented, subsequently the significant parameters of dehazing scheme using the atmospheric degradation model are challenging to achieve in the environments of nighttime imaging. In addition, the significant model parameters of guided image filter are nominated in correspondence to the boundary information of initial image instead of the initial image itself, which makes it more beneficial for dehazing image acquired under night time environments.

\section{Proposed Scheme}

This section introduces the overall framework of the proposed night-time single image dehazing scheme. From the illustration Figure 1 describes the processing pipeline of the entire proposed scheme. The proposed scheme is initiated by 


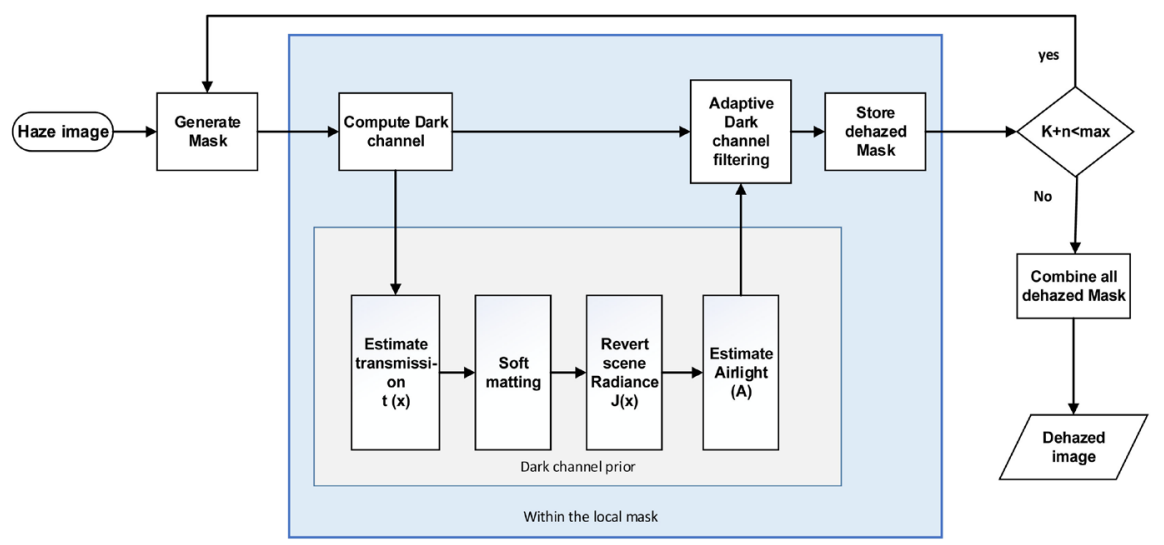

Figure 1. An illustration of the overall framework of the proposed scheme.

generating mask on a given hazed image. Our proposed technique is based on local dark channel dehazing. The procedure extensively establishes adaptive filtering technique in the estimation and improving dehazing effects that are applied on the depth paths across a selected local mask on hazed image. In this approach depth information is taken into consideration since it is a core element for guiding the procedure. The proposed method is capable of estimating the atmospheric intensities within a local mask of a given haze image for enhancement.

Additionally, it is capable of overcoming the challenge of night-scenes that is considered to inherit artificial and spatially non-uniform environmental illumination. The proposed scheme estimates the atmospheric light locally within a selected mask on the hazy image independently. We leveraged the adaptive filtering capacity of guided filter [23] to acquire a fixed value $\mu_{x}$ at each local region centered at $x$. More specifically, we partitioned the image into a grid of cells, each cell with a size $l \times w$. Then, guided filtering with a filter size of $3 \times 3$ was performed on each grid with a stepping value $n$. Following this, we estimate the pixels within local mask of hazy image that fall within the range target to be attributed to haze pixels. The pixels estimated in the dehazed masks are incremented by the value of 1 at each iteration for each mask. The scheme performs an adaptive dark channel filtering on the reconstructed mask and stored in an iterative manner. Applying the acquired estimated atmospheric intensity map, we estimate the transmission through the dark channel prior, this can be expressed as:

$$
t(x)=1-\min _{y \in \Omega(x)}\left(\min _{c} \frac{I^{c}(x)}{A^{c}(x)}\right)
$$

where $\Omega$ denotes small patch, and $x$ is the location index inside the patch. The upper RGB threshold is represented as max (RGB). Based on the step value $n$, we ensured that $k+n$ is below the max threshold values. Whereas this condition tends to be true and all iterations are completed. The scheme continues with a mask combination operation for all the reconstructed masks of the night time haze image to produce an enhanced image under nighttime environment. Moreover, the reconstructed mask has the advantage of estimating the atmospheric in- 
tensity evenly across the local mask. Hence the combined mask operation yields an enhanced reconstructed and uniform image under night time environment.

\section{Experimental Verification and Evaluation Results}

This section discusses the evaluation and the verification of various dehazing methods. In image dehazing, it is mostly difficult to evaluate the performance and efficiency simply by human vision. For this reason, it is essential to compile all the characters of the various dehazing algorithms and analyze them in general. To ensure impartiality, all the corresponding algorithms are realized on the same matlab platform. Qualitative visual Assessment is a subjective approach based on the visual qualities of dehazed images whiles Quantitative Experimental Evaluation represents an objective assessment approach based on intrinsic properties or metrics.

\section{1) Qualitative Visual Evaluation}

In this section, we discuss the qualitative visual assessment of the proposed scheme which performs efficiently in restoring the visual quality of the image within the RGB channels. Besides, the scheme is capable of improving the depth information within the scene is significantly. As illustrated in Figure 2 below where (a) is the input haze image (b) is the depth map of the hazy scene (c) is the dehazed image and (d) represents the enhanced depth map of the dehazed scene. Based on human observation of these images with their corresponding depth maps, the refined image inherits good quality with a bright corresponding depth map as compared to the hazy image and its depth map. Furthermore, by

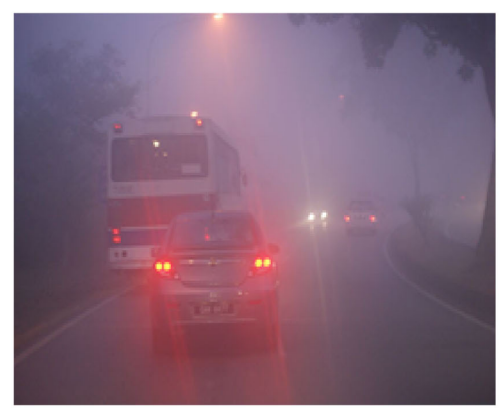

(a)

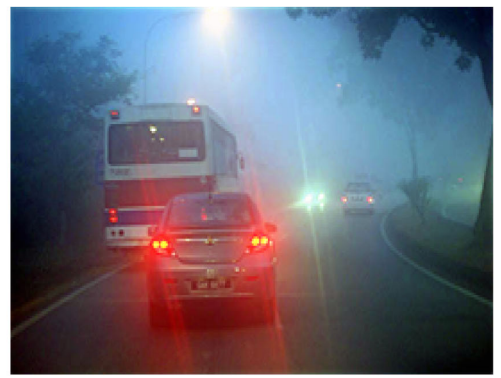

(c)

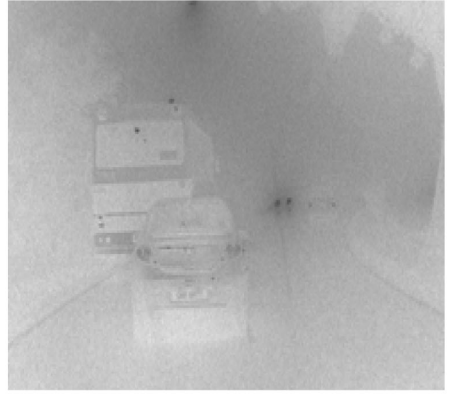

(b)

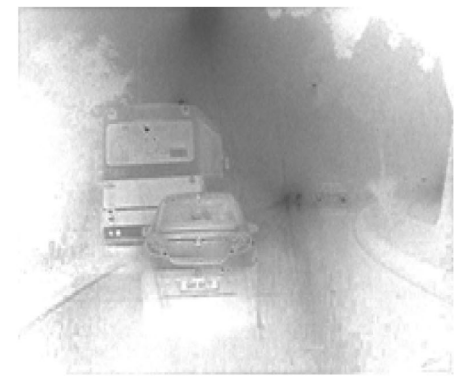

(d)

Figure 2. The proposed dehazing scheme effectively reconstructs the scene depth, essentially restoration depth information within the scene (a) Haze input image; (b) Depth-map of haze image; (c) Dehazed image; (d) Transmission map of dehazed image. 
employing a local methodology to address night-time dehazing, the scheme's efficiency is not limited to nearby patches within the image but similar efficiency is homogeneously extended to distant regions within the image.

Figure 3 presents the visual qualitative comparison results of the proposed method and corresponding state of the art, where (a) denotes the haze image input (b) represents He et al. [3] (c) represents Li et al. [20], (d) represents Meng et al. [22], (e) represents Zhang et al. [21] and (f) presents our proposed scheme. We discuss and highlight on the visual qualities between proposed scheme and the corresponding state of the art image dehazing methods which is based on the visual perception of the refined image by human observation. From Figure 3, the output of (b) He et al. appears to inherit dim properties associated with halos in the dehazed image. The output of (c) Li et al. is quite substantial. However, it is associated with color distortions and presence of haze on the edges of the dehazed image. The output of (d) Meng et al. [22] presents astonishing results however the restored image inherits an overestimation quality, which results in color infidelities. The output of (d) presents is quite reasonable however recovered Image appears to have low illumination and unnatural colors. Finally, the output of (f) the proposed scheme is astonishing which gains superiority over most of the state of the art. However, for high depth ranges dehazing results are only effective for close-ranged patches while a significant volume of haze continues to remain in distant regions. This drawback associated with the lack of effective
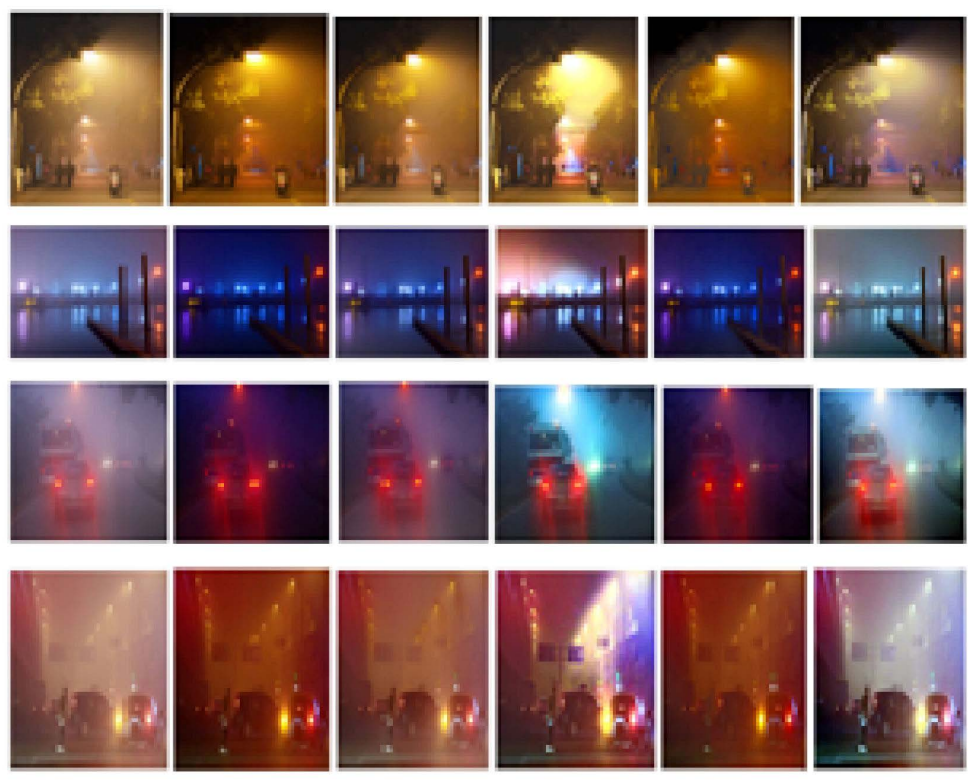

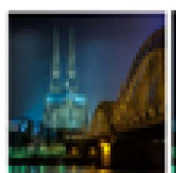

(a)

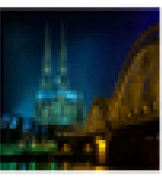

(b)

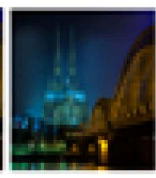

(c)

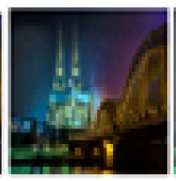

(d)

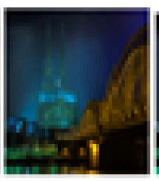

(e)

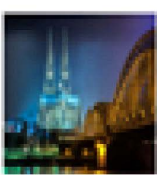

(f)

Figure 3. Visual comparison of various state-of-the-art dehazing algorithms with the proposed method. 
depth-modeling strategies which are capable of intuitively adjusting parameters for the numerous depth patches within the image. This has numerous adverse effects on higher level machine vision or learning schemes which may only rely on single images from the scene for the realization of feature extraction. Finally, due to the stringency in filtering that is applied in some of the state-of-the-art, some results may end up with smoothing effects that may remove certain crucial edge and boundary features. In contrast with the state-of-the-art, the proposed method achieves results with high levels of visual clarity and color fidelity.

\section{2) Quantitative Experimental Evaluation}

In this section of the paper, we conduct as well as discuss an objective evaluation procedure. This is used to evaluate quantitative data based on their corresponding objective metrics. On the contrary to the previous section above where we highlight the visual properties and comparisons of various state of the art dehazing algorithms based on visual opinion of a user subjectively. However, it is not an adequate assessment to be concluded on. Since image dehazing algorithms are not constrained to only image restoration but also very potential in the improvement of the intrinsic properties of the restored image. For this reason, in order to determine the performances of intrinsic properties of the output images of various algorithms, the intrinsic metrics used to compare and evaluate quantitative data comprises Mean Squared Error (MSE), Peak Signal to Noise Ratio (PSNR), Signal to Noise Ratio (SNR) and Structural Similarity Index Measure (SSIM) respectively. Nonetheless, the cognitive challenge of objective evaluation or quantitative assessment is the unavailability of reference image. Since the computation of previously stated metrics demands the both the original image and reference image before the corruption of haze. The tables below represent the objective metrics performances as well as comparisons of the selected state-of-the-art of unconstraint night time haze removal procedures.

The quantitative results presented in Table 1 emphasize on the performances and the effectiveness of the selected dehazing procedures under night-time hazy conditions by assessing the recovered images based on their intrinsic image properties respectively. Our proposed scheme distinctively achieves superiority and an excellent results over the selected state-of-the-art on the MSE and the PSNR metric indicated in bold, since its capable of achieving less mean squared error and a better peak signal-to-noise ratio than the state-of-the-art, also the proposed scheme is capable of achieving a satisfactory results with the signal-to-noise ratio and structural similarity index measure (SSIM) metric over the existing methods. Additionally, we take into consideration real-time system requirements of these methods which makes it needful to address the computational complexities of dehazing approaches. We establish comparisons of the state-of-the-art and the proposed scheme based on computational complexity in Table 2 below. From the results achieved indicates that zhang et al. [21] attains superiority over all the experimented approaches followed by our proposed scheme which attains impressive computational speeds at the second place over the other state-of-the-art. 
Table 1. A comparison of the proposed algorithm with state-of-the-art using intrinsic image properties.

\begin{tabular}{ccccc}
\hline Algorithms & MSE & PSNR & SNR & SSIM \\
\hline He et al. [3] & 3787.36 & 13.44 & 5.82 & 0.65 \\
Li et al. [20] & 1079.22 & 18.68 & 11.01 & 0.93 \\
Zhang et al. [21] & 3442.3 & 14.01 & 6.39 & 0.76 \\
Meng et al. [22] & 1327.99 & 18.17 & 10.49 & 0.71 \\
Proposed Scheme & $\mathbf{9 6 0 . 8 4}$ & $\mathbf{1 9 . 4 5 6}$ & $\mathbf{1 0 . 6 8}$ & $\mathbf{0 . 8 4}$ \\
\hline
\end{tabular}

Table 2. A comparison of the proposed algorithm with state-of-the-art based on computational time.

\begin{tabular}{cc}
\hline Algorithms & Time/s \\
\hline He et al. [3] & 436.61 \\
Li et al. [20] & 1079.22 \\
Zhang et al. [21] & 6.11 \\
Meng et al. [22] & 124.76 \\
Proposed Scheme & $\mathbf{5 0 . 6 3}$ \\
\hline
\end{tabular}

\section{Conclusion}

The work in this paper emphasizes an efficient and attractive single image haze removal procedure which is capable of image restoration under night time hazy environment. Our proposed scheme initiates by generating masks in a given night time hazy image, inspired by dark channel prior which is capable of estimating depth information and restoring depth features within a local mask of a target haze image. The approach takes into consideration of the non-uniform illumination which is due to the presence of an artificial light source or multiple light sources in images captured under night environment. Besides, the proposed scheme addresses haze effects due to scattering and attenuation of light. Since the proposed scheme is performed on a local mask iteratively on a given haze image. In addition, experimental verifications and comparisons were conducted on the proposed scheme and the state-of-the-art. In the experimental qualitative analysis, the proposed scheme achieves superiority in terms of clarity and vividness of image over the selected state-of-the-art. Similarly, with the quantitative experimental analysis, the proposed scheme distinctively achieves superiority over the selected state-ofthe-art on the MSE and the PSNR metric. Subsequently, it's capable of achieving less mean squared error as well as a better peak signal-to-noise ratio than the stateof-the-art. Besides, the proposed scheme has the advantage of achieving a satisfactory result with the signal-to-noise ratio and structural similarity index measure (SSIM) metric over the existing methods. Our proposed scheme demonstrates impressive results in terms of computational speeds over the state-of-the-art. Comparatively, the proposed scheme achieves less computational complexities, which makes it more feasible in real time domain than the selected state-of-art. 


\section{Acknowledgements}

The authors would like to express their appreciation to the 303 research lab of the School of Automation Science and Engineering, South China University of Technology. This work was supported by Guangdong Provincial Science and Technology Major Project (2018B010108001).

\section{Conflicts of Interest}

The authors declare no conflicts of interest regarding the publication of this paper.

\section{References}

[1] Nayar, S.K. and Narasimhan, S.G. (1999) Vision in Bad Weather. Proceedings of the Seventh IEEE International Conference on Computer Vision, 2, 820-827. https://doi.org/10.1109/ICCV.1999.790306

[2] Tan, R.T. (2008) Visibility in Bad Weather from a Single Image. 2008 IEEE Conference on Computer Vision and Pattern Recognition, Anchorage, AK, 23-28 June 2008, 1-8. https://doi.org/10.1109/CVPR.2008.4587643

[3] He, K., Sun, J. and Tang, X. (2009) Single Image Haze Removal Using Dark Channel Prior. 2009 IEEE Conference on Computer Vision and Pattern Recognition, Miami, FL, 20-25 June 2009, 1956-1963. https://doi.org/10.1109/CVPRW.2009.5206515

[4] Fattal, R. (2008) Single Image Dehazing. ACM Transactions on Graphics, 27, Article No. 72. https://doi.org/10.1145/1360612.1360671

[5] Huang, S.C. (2011) An Advanced Motion Detection Algorithm with Video Quality Analysis for Video Surveillance Systems. IEEE Transactions on Circuits and Systems for Video Technology, 21, 1-14. https://doi.org/10.1109/TCSVT.2010.2087812

[6] Negru, M., Nedevschi, S. and Peter, R.I. (2015) Exponential Contrast Restoration in Fog Conditions for Driving Assistance Intelligent Transportation Systems. IEEE Transactions, 16, 2257-2268. https://doi.org/10.1109/TITS.2015.2405013

[7] Halmaoui, H., Cord, A. and Hautiere, N. (2011) Contrast Restoration of Road Images Taken in Foggy Weather. 2011 IEEE International Conference on Computer Vision Workshops, Barcelona, Spain, 6-13 November 2011, 2057-2063. https://doi.org/10.1109/ICCVW.2011.6130501

[8] Schechner, Y.Y., Narasimhan, S.G. and Nayar, S.K. (2001) Instant Dehazing of Images Using Polarization. Proceedings of the 2001 IEEE Computer Society Conference on Computer Vision and Pattern Recognition, 1, 325-332.

[9] Narasimhan, S.G. and Nayar, S.K. (2003) Contrast Restoration of Weather Degraded Images. IEEE Transactions on Pattern Analysis and Machine Intelligence, 25, 713-724. https://doi.org/10.1109/TPAMI.2003.1201821

[10] Narasimhan, S.G. and Nayar, S.K. (2002) Vision and the Atmosphere. International Journal of Computer Vision, 48, 233-254. https://doi.org/10.1023/A:1016328200723

[11] McCartney, E.J. (1976) Optics of the Atmosphere: Scattering by Molecules and Particles. Volume 1, John Wiley and Sons Inc., New York.

[12] Tang, K., Yang, J. and Wang, J. (2014) Investigating Haze-Relevant Features in a Learning Framework for Image Dehazing. 2014 IEEE Conference on Computer Vision and Pattern Recognition, Columbus, OH, 23-28 June 2014, 2995-3002. https://doi.org/10.1109/CVPR.2014.383 
[13] Tarel, J.P. and Hautiere, N. (2009) Fast Visibility Restoration from a Single Color or Gray Level Image. 2009 IEEE 12th International Conference on Computer Vision, Kyoto, Japan, 29 September-2 October 2009, 2201-2208. https://doi.org/10.1109/ICCV.2009.5459251

[14] Gibson, K., VO, D. and Nguyen, T. (2010) An Investigation in Dehazing Compressed Images and Video. OCEANS 2010 MTS/IEEE SEATTLE, Seattle, WA, 20-23 September 2010, 1-8. https://doi.org/10.1109/OCEANS.2010.5664479

[15] Liu, Q., Chen, M. and Zhou, D. (2013) Fast Haze Removal from a Single Image. Proceedings of the 25th Chinese Control and Decision Conference, Guiyang, 25-27 May 2013, 3780-3785. https://doi.org/10.1109/CCDC.2013.6561607

[16] Land, E.H. (1977) The Retinex Theory of Color Vision. Scientific American, 237, 108-128. https://doi.org/10.1038/scientificamerican1277-108

[17] Jobson, D.J., Rahman, Z. and Woodell, G.A. (1997) Properties and Performance of a Center/Surround Retinex. IEEE Transactions on Image Processing, 6, 451-462. https://doi.org/10.1109/83.557356

[18] Jobson, D.J., Rahman, Z. and Woodell, G.A. (1997) A Multiscale Retinex for Bridging the Gap between Color Images and the Human Observation of Scenes. IEEE Transactions on Image Processing, 6, 965-976. https://doi.org/10.1109/83.597272

[19] Pei, S.C. and Lee, T.Y. (2012) Nighttime Haze Removal Using Color Transfer PreProcessing and Dark Channel Prior. 2012 19th IEEE International Conference on Image Processing, Orlando, FL, 30 September-3 October 2012, 957-960. https://doi.org/10.1109/ICIP.2012.6467020

[20] Li, Y., Tan, R.T. and Brown, M.S. (2015) Nighttime Haze Removal with Glow and Multiple Light Colors. 2015 IEEE International Conference on Computer Vision, Santiago, Chile, 7-13 December 2015, 226-234.

[21] Zhang, J., Cao, Y. and Wang, Z. (2014) Nighttime Haze Removal Based on a New Imaging Model. 2014 IEEE International Conference on Image Processing, Paris, France, 27-30 October 2014, 4557-4561. https://doi.org/10.1109/ICIP.2014.7025924

[22] Jiang, B., Meng, H., Ma, X., Wang, L., Zhou, Y., Xu, P.F., Jiang, S.Y. and Meng, X.J. (2018) Nighttime Image Dehazing with Modified Models of Color Transfer and Guided Image Filter. Multimedia Tools and Applications, 77, 3125-3141. https://doi.org/10.1007/s11042-017-4954-9

[23] He, K., Sun, J. and Tang, X. (2013) Guided Image Filtering. IEEE Transactions on Pattern Analysis and Machine Intelligence, 35, 1397-1409. https://doi.org/10.1109/TPAMI.2012.213 